\title{
Downregulation of annexin A3 inhibits tumor metastasis and decreases drug resistance in breast cancer
}

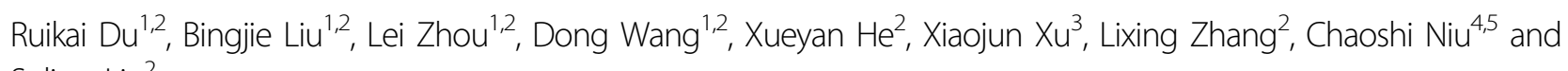
Suling $\mathrm{Liu}^{2}$

\begin{abstract}
Annexin A3 (ANXA3) is dysregulated and plays an important role in various cancers. However, the role of ANXA3 in breast cancer is still unclear. Here, we observed that the expression level of ANXA3 was significantly upregulated in breast cancer tissues. ANXA3 knockdown inhibited cell invasion but promoted cell proliferation in both in vitro and in vivo assays. Furthermore, we found that ANXA3 knockdown inhibited the NFKB pathway via upregulating IkBa, resulting in mesenchymal-epithelial transition (MET) and a heterogeneity change of breast cancer stem cells (BCSCs). In addition, we demonstrated that ANXA3 knockdown increased the sensitivity of breast cancer cells to doxorubicin by increasing the drug uptake. The combination of ANXA3 knockdown and doxorubicin treatment simultaneously inhibited tumor growth and metastasis in vivo. This study described the role and mechanisms of ANXA3 in regulating BCSCs and breast cancer growth and metastasis, indicating that downregulating ANXA3 together with chemotherapy might be a novel therapeutic strategy for treating breast cancer.
\end{abstract}

\section{Introduction}

Breast cancer is the most common malignancy in women and is a serious threat to women's health ${ }^{1}$. Similar to other cancers, in breast cancer, metastasis accounts for the vast majority of breast cancer deaths ${ }^{2}$. Despite advances in cancer diagnosis and treatment in recent years, traditional treatments (radiotherapy, chemotherapy, and hormone therapy) are always limited by the resistance of some tumor cells ${ }^{3}$, thus forcing researchers to continue to look for new therapeutic approaches and targets.

\footnotetext{
Correspondence: Chaoshi Niu (niuchaoshi@163.com) or

Suling Liu (suling@fudan.edu.cn)

${ }^{1}$ The CAS Key Laboratory of Innate Immunity and Chronic Disease, Hefei National Laboratory for Physical Sciences at the Microscale, School of Life Science and Medical Center, University of Science \& Technology of China, Hefei, Anhui 230027, China

${ }^{2}$ Key Laboratory of Breast Cancer in Shanghai, Cancer Institute, Department of Breast Surgery; Institutes of Biomedical Sciences; Innovation Center for Cell Signaling Network; Fudan University Shanghai Cancer Center, Shanghai 200032, China

Full list of author information is available at the end of the article

Ruikai Du and Bingjie Liu contributed equally to this work.

Edited by $Y$. Shi
}

Epithelial-mesenchymal transition (EMT) is a process of epithelial cells losing their cell polarity and cell adhesion and acquiring invasive properties to become mesenchymal cells. E-cadherin and Vimentin are considered markers of epithelial cells and mesenchymal cells, respectively ${ }^{4}$. EMT is necessary for the development of embryos $^{5}$ and has been proven to play an important role in tumor metastasis and drug resistance ${ }^{6,7}$. Several important signaling pathways (Wnt/ $\beta$-catenin, MAPK and NF- $\mathrm{\kappa B}$ ) are involved in EMT and correlated with tumor progression $^{8-10}$.

In recent years, the cancer stem cell (CSC) hypothesis has been proposed, suggesting that CSCs play a decisive role in the development and progression of multiple cancers ${ }^{11-14}$ and are responsible for the recurrence of cancer due to their strong tolerance to traditional chemotherapies ${ }^{15}$. Therefore, clarifying the regulation mechanisms of CSCs is critical for developing more effective therapies for cancers. Our previous research showed breast cancer stem cells (BCSCs) have heterogeneity and exist in a distinct, invasive, 
mesenchymal-like state marked by $\mathrm{CD} 24^{-} / \mathrm{CD} 44^{+}$and a proliferative, epithelial-like state marked by high aldehyde dehydrogenase activity $\left(\mathrm{ALDH}^{+}\right)^{16}$. Despite our growing understanding of the importance of and complexity of BCSCs, the mechanisms of BCSC regulation remain limited.

Annexin A3 (ANXA3) is a member of the annexin family, which can bind to acidic phospholipids in a calcium-dependent manner ${ }^{17}$. ANXA3 has a role in cell differentiation, cell migration, immune regulation, and bone formation ${ }^{18}$. In recent years, it has been shown that ANXA3 plays a role in a variety of tumor development processes. Overexpression of ANXA3 promotes tumor proliferation and metastasis in lung, liver, and ovarian carcinoma ${ }^{19-22}$ and is associated with chemotherapy resistance ${ }^{22-24}$. The latest research shows that ANXA3 is highly expressed in $\mathrm{CD} 133^{+}$liver CSCs and plays a regulatory function ${ }^{25,26}$. However, the function of ANXA3 in breast cancer remains to be elucidated, and the effect of ANXA3 on regulating BCSCs has not been investigated.

In this study, we found that ANXA3 is significantly upregulated in breast tumor tissues from clinical biopsies. ANXA3 knockdown suppressed breast cancer cell invasion but promoted proliferation both in vitro and in vivo, which was due to the IкB $\alpha$-mediated mesenchymal-epithelial transition and the switch of different states of BCSCs. In addition, we also showed that ANXA3 knockdown promoted the uptake of doxorubicin, and the inhibition of ANXA3 in combination with doxorubicin could efficiently lead to blocking tumor growth as well as tumor metastasis.

\section{Results}

ANXA3 is upregulated in breast cancer tissues and is positively correlated with poor overall survival

To determine the expression pattern of ANXA3 in breast cancer samples, 16 pairs of breast cancer tissues and their corresponding adjacent normal tissues were analyzed for $A N X A 3$ expression using quantitative reverse transcription quantitative real-time PCR (qRT-PCR). The results showed that $A N X A 3$ was significantly upregulated in tumor tissues (Fig. 1a). At the same time, the protein level of ANXA3 was verified by immunohistochemistry (IHC; Fig. 1b). To further investigate the correlation between ANXA3 expression and the patients' overall survival, we analyzed data from 471 patients in the Oncomine database. Kaplan-Meier analysis indicated that patients with low ANXA3 expression had a longer overall survival (Fig. 1c). These results suggest that upregulation of ANXA3 is associated with poor prognosis in breast cancer, indicating the ANXA3 might be a good predictor of prognosis for breast cancer patients.

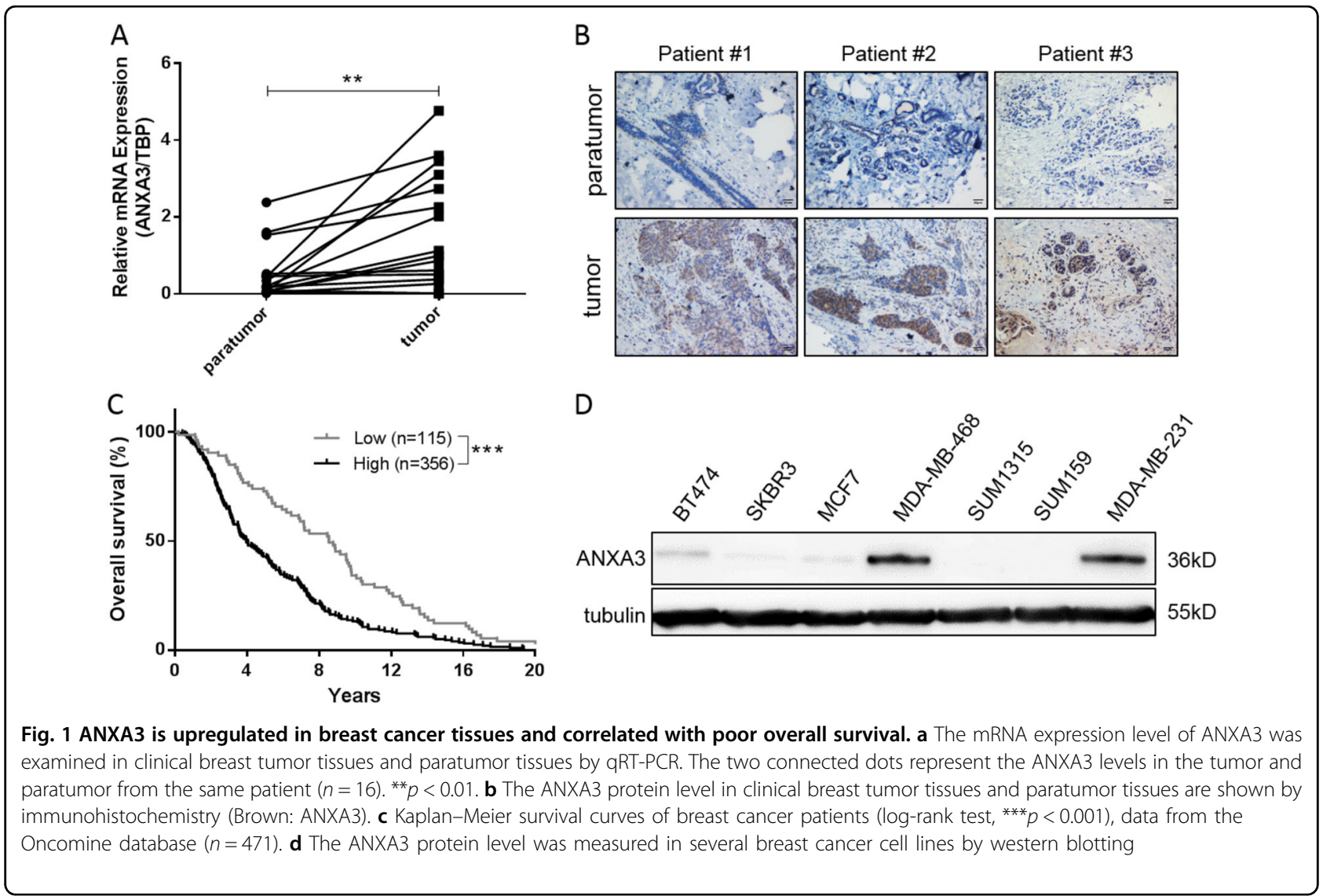


ANXA3 knockdown inhibits cell invasion but promotes cell proliferation in vitro via inducing mesenchymal-epithelial transition

To examine the function of $A N X A 3$ in breast cancer, we analyzed $A N X A 3$ expression in different human breast cancer cell lines by western blot. We showed that MDAMB-231 and MDA-MB-468 had markedly higher ANXA3 protein levels (Fig. 1d). To explore the effect of $A N X A 3$ on breast cancer cells, we established $A N X A 3-$ knockdown cell lines using MDA-MB-231, MDA-MB-468, and mouse mammary cancer cell line 4T1. Each cell line was infected by lentivirus with two specific short hairpin RNA (shRNA) sequence which targeted ANXA3 mRNA and a random sequence shRNA as the control. Both of the specific shRNAs significantly decrease the ANXA3 expression level (Fig. 2a). In the Matrigel transwell assay, $A N X A 3$ knockdown inhibited cell invasion significantly (Fig. 2b, c). Interestingly, utilizing the MTT cell proliferation assay, we showed that $A N X A 3$ knockdown accelerated cell proliferation significantly (Fig. 2d). Our previous study demonstrates that in breast tumors, mesenchymal-like BCSCs are relatively quiescent and have a highly invasive capacity, whereas the epithelial-like
BCSCs are more proliferative ${ }^{16}$. To determine whether ANXA3 knockdown induced mesenchymal-epithelial transition (MET), we analyzed the expression of epithelial markers E-cadherin and $\gamma$-catenin and mesenchymal markers Vimentin and N-cadherin by western blotting. Those results showed that $A N X A 3$ knockdown decreased mesenchymal marker expression but increased epithelial marker expression (Fig. 2e), which suggested that $A N X A 3$ knockdown induced the MET process in breast cancer cell lines.

\section{ANXA3 knockdown inhibits metastasis but promotes tumor growth in vivo}

The in vitro studies above showed that ANXA3 knockdown could significantly inhibit cell invasion but promote tumor cell proliferation. To further verify those results in vivo, 50,000 MDA-MB-231 cells with or without $A N X A 3$ knockdown were implanted into the mammary glands of 6- to 8-week-old female nude mice. The tumor size was monitored and measured weekly, and the results showed that $A N X A 3$ knockdown could significantly promote tumor growth (Fig. 3a). After the mice were sacrificed, tumors were harvested, photographed, and weighed.

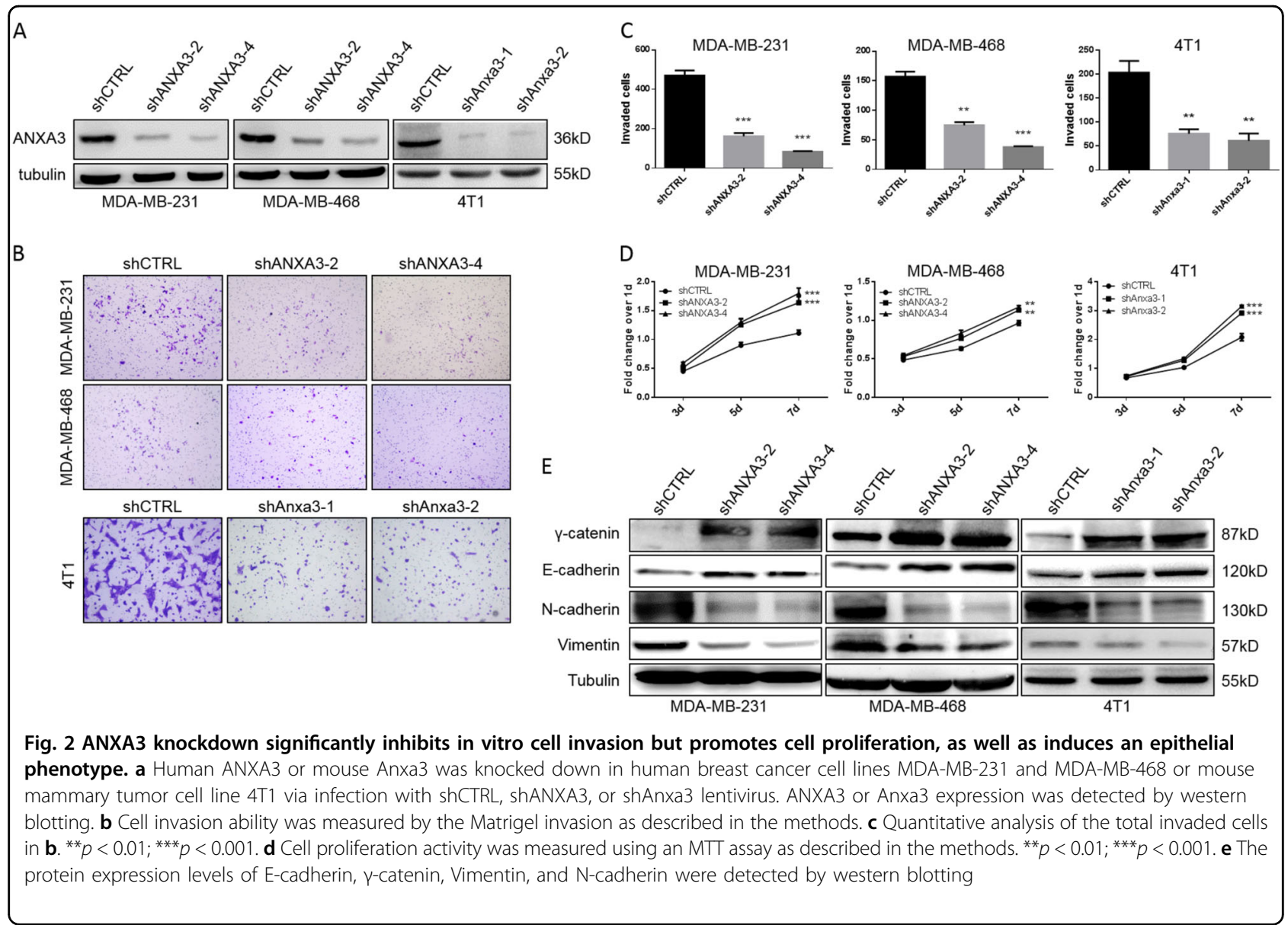




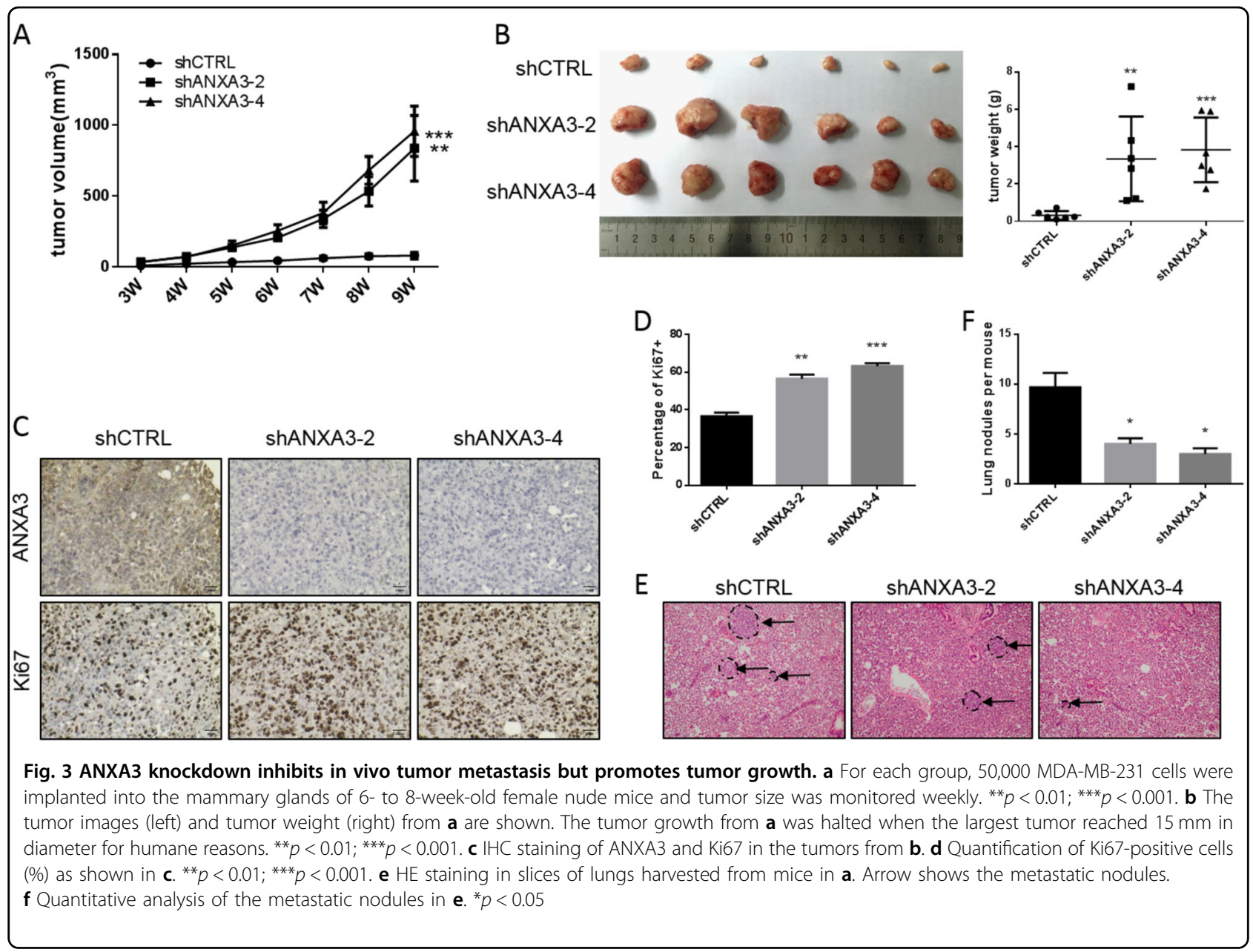

The tumors from the $A N X A 3$ knockdown group were significantly larger and heavier than those from the control group (Fig. $3 \mathrm{~b}$ ). Then, we stained the tumor sections for ANXA3 and Ki67 utilizing IHC staining and showed that ANXA3 was indeed downregulated in the ANXA3knockdown group, and that $A N X A 3$ knockdown was correlated with much higher Ki67 expression, which characterizes cell proliferation activity (Fig. 3c, d). At the same time, lung slices of those mice were also subjected to hematoxylin-eosin (HE) staining, and the results showed that there were fewer metastatic nodules in the ANXA3knockdown group (Fig. 3e, f). These in vivo results suggested that ANXA3 knockdown promoted breast cancer tumor growth but inhibited metastasis.

\section{ANXA3 inversely regulates two different states of breast cancer stem cells}

Based on previous studies, BCSCs play a key role in tumor initiation and recurrence ${ }^{27}$. Our previous study showed that BCSCs exist in two alternative states: mesenchymal-like state BCSCs $\left(\mathrm{CD} 24^{-} / \mathrm{CD} 44^{+}\right)$, which are relatively quiescent and have highly invasive capacity, and epithelial-like state $\mathrm{BCSCs}\left(\mathrm{ALDH}^{+}\right)$, which are associated with extensive proliferation ${ }^{16}$.

To further characterize the role of $A N X A 3$ in BCSC regulation, we used two sets of markers for cell sorting by flow cytometry to obtain different subgroups of BCSCs in the MDA-MB-231 cell line. Then, we used qRT-PCR to detect the expression pattern of $A N X A 3$ in different subgroups of BCSCs (Fig. 4a). ANXA3 showed a distinct expression pattern between the different subgroups. The expression of $A N X A 3$ was higher in the mesenchymal-like BCSCs (the CD24 ${ }^{-} / \mathrm{CD} 44^{+}$population versus the non$\mathrm{CD} 24^{-} / \mathrm{CD} 44^{+}$population) but lower in the epithelial-like state (the $\mathrm{ALDH}^{+}$population versus the $\mathrm{ALDH}^{-}$population). Next, we examined the proportion of each BCSC population in the ANXA3-knockdown cell line, and we found that the $\mathrm{CD} 24^{-} / \mathrm{CD} 44^{+}$population was decreased, but the $\mathrm{ALDH}^{+}$population was increased (Fig. 4b, c, and S1). Similar findings were observed in the tumors from Fig. 3 (Fig. 4d). Taken together, these results suggest that ANXA3 knockdown decreases the highly metastatic 


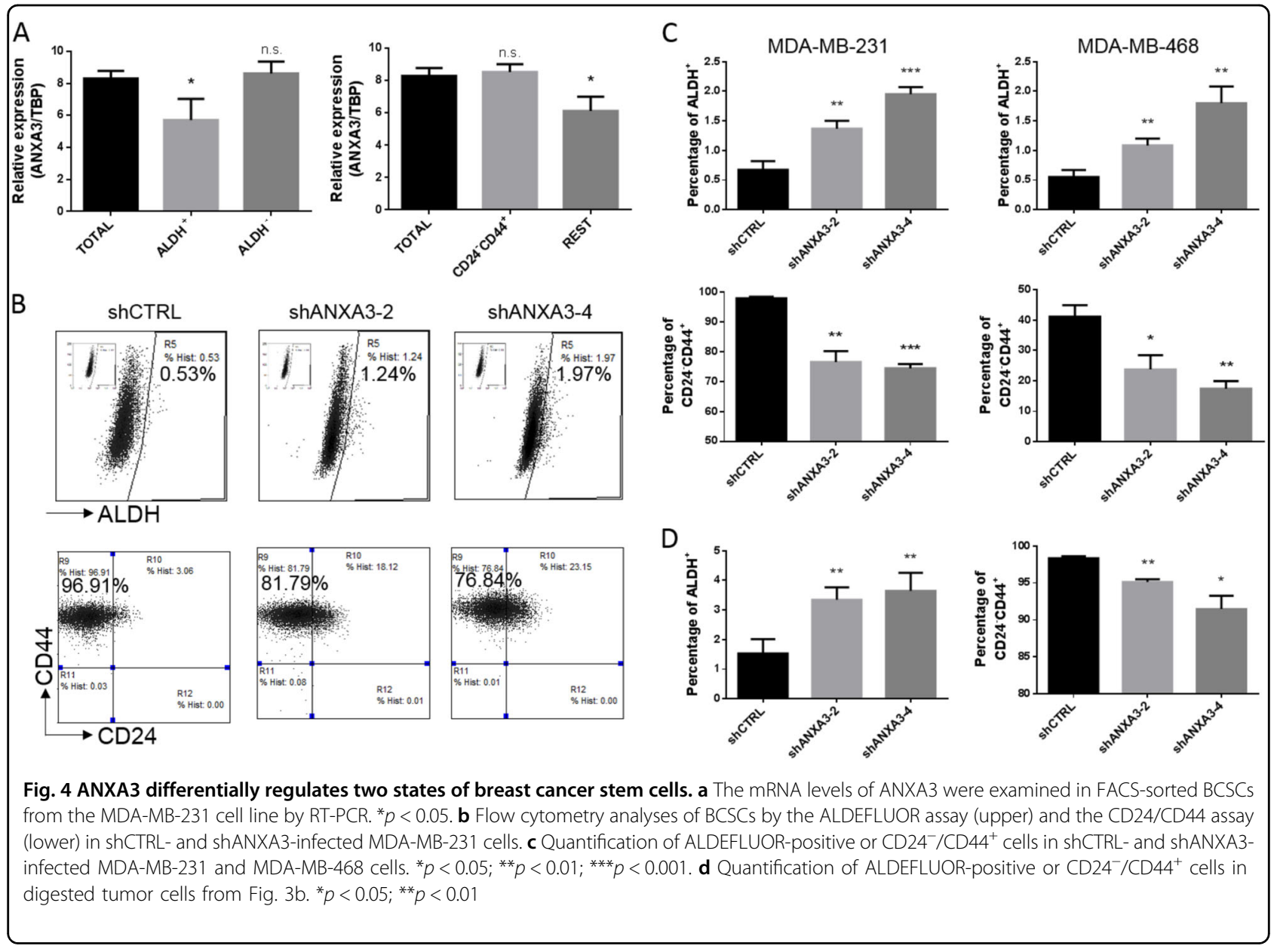

mesenchymal-like BCSCs but enriches the highly proliferative epithelial-like BCSCs, which might explain why the ANXA3 knockdown inhibited cancer cell invasion and tumor metastasis but promoted cancer cell proliferation and tumor growth.

\section{The NF-KB signaling pathway plays a key role in} ANXA3-regulated cancer cell proliferation and invasion

To reveal the mechanisms of $A N X A 3$ knockdowninduced MET, ANXA3-knockdown and control cell lines were collected, and RNA-Seq was performed. Heatmap analysis showed that gene expression patterns are different between the control and ANXA3-knockdown groups (Fig. 5a). A scatter chart of the top ten enrichment pathways is shown (Fig. 5b), which suggests numbers of genes in the NF- $\mathrm{kB}$ signaling pathway were enriched in the profiling data. In addition, the mRNA expression of several significantly changed genes was confirmed by qRT-PCR (Fig. 5c). Considering IкB $\alpha$ (encoded by the NFKBIA gene) is a cellular protein that functions to inhibit the NF- $\kappa B$ transcription factor ${ }^{28}$, I $\kappa \mathrm{B} \alpha$ and phospho-NF- $\kappa \mathrm{B}$ p65, an indicator of the activated canonical NF- $\mathrm{KB}$ pathway ${ }^{29}$, were analyzed by immunoblotting to validate NF- $\mathrm{kB}$ signaling pathway alteration (Fig. 5d). Increased IкB $\alpha$ and reduced pp65 in the ANXA3-knockdown cells vs. scramble shRNA control suggested that the ANXA3 knockdown inhibited the NF- $\kappa B$ pathway. To further investigate whether the NF- $\mathrm{kB}$ pathway plays a key role in the $A N X A 3$ knockdowninduced MET process, IкB $\alpha$-knockdown cell lines with or without the ANXA3 knockdown were established in the MDA-MB-231 cell line via a specific shRNA lentivirus infection. IкB $\alpha, \mathrm{p}-\mathrm{p} 65$, and mesenchymal/epithelial markers Vimentin/E-cadherin were detected by western blotting (Fig. 5e). The results showed that the MET state induced by the $A N X A 3$ knockdown was attenuated to some degree by the IкB $\alpha$ knockdown. The cell proliferation assay and cell invasion assay revealed that double knockdown of $A N X A 3$ and IкB $\alpha$ reduced cell proliferation and increased invasion compared to the ANXA3 knockdown alone (Fig. 5f, h). Furthermore, the IкB $\alpha$ knockdown alone has little effect on either state of the BCSCs, but the IкB $\alpha$ knockdown partially abrogated the effects of the ANXA3 knockdown on both the $\mathrm{CD} 24^{-} / \mathrm{CD} 44^{+}$population and the $\mathrm{ALDH}^{+}$population (Fig. $5 \mathrm{i}$ and S2). These results 


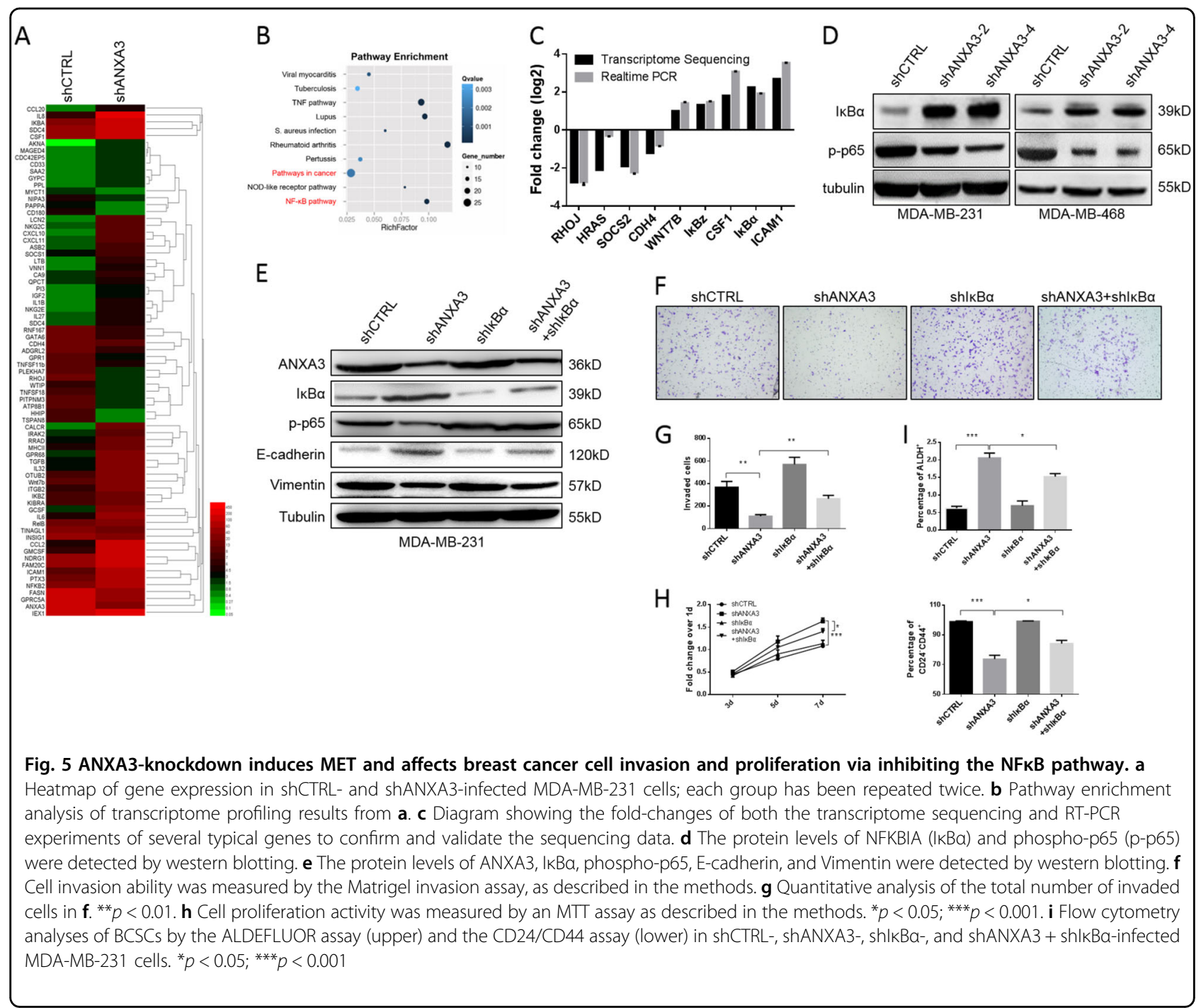

suggest that the NF- $\mathrm{BB}$ pathway played an important role in the $A N X A 3$ knockdown-induced MET process, in cancer cell proliferation and in cell invasion, and the $\operatorname{I\kappa B} \alpha$ knockdown abrogated the phenomenon caused by the ANXA3 knockdown.

\section{The ANXA3 knockdown enhances the efficacy of} doxorubicin on tumor growth by increasing the uptake of doxorubicin in breast cancer cells

As recent studies have shown that high $A N X A 3$ expression is involved in resistance to chemotherapy reagents in several cancers ${ }^{21,22,24}$, we investigated the effect of $A N X A 3$ on chemotherapy sensitivity in breast cancer cell lines. It has been shown that $A N X A 3$ reduced the accumulation of platinum in ovarian cancer cells ${ }^{22}$. Here, we analyzed the uptake of doxorubicin (Dox), which is widely used in breast cancer treatment, in ANXA3knockdown cell lines by flow cytometry (Fig. 6a). Both human breast cancer cell line MDA-MB-231 and mouse mammary cancer cell line 4T1 showed an increased cellular accumulation of Dox in ANXA3-knockdown cells. Furthermore, we showed $A N X A 3$-knockdown enhance the efficacy of both doxorubicin and docetaxel as the result of NF- $\kappa$ B pathway inhibition (Fig. S3A, B). Next, to ascertain whether the $A N X A 3$ knockdown influenced the sensitivity of breast cancer cells to Dox in vivo, the 4T1 tumor model was utilized. shCTRL cells and Anxa3 knockdown 4T1 cells were implanted to the fourth mammary glands of BALB/c mice, and each group was divided into two groups for different treatments when the tumor size reached approximately $2-3 \mathrm{~mm}$ in diameter: one group was treated with saline (control) and the other group was treated with Dox. After 4 weekly treatments, mice were sacrificed, and tumors were photographed and metastatic nodules in the lungs were counted. The results showed that $A N X A 3$ knockdown promoted breast tumor 


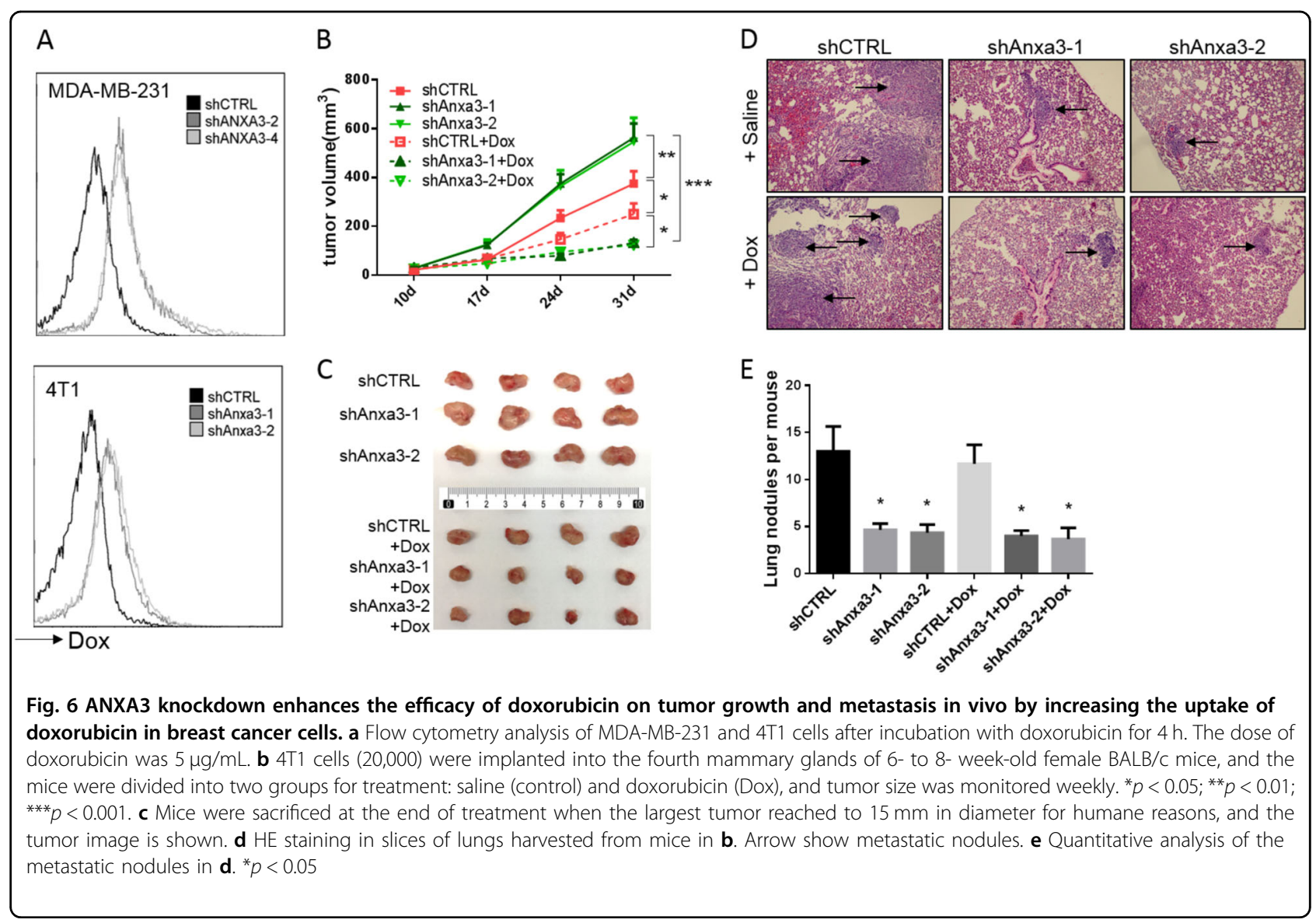

growth (Fig. 6b,c). However, tumor metastasis was reduced (Fig. 6d), as we have observed in the human breast cancer cell line xenografts. Interestingly, $A N X A 3$ knockdown enhanced the sensitivity of the cells to Dox, which antagonized the tumor-promoting effect from the $A N X A 3$ knockdown. And it has been reported that $\mathrm{CD} 24^{-} / \mathrm{CD} 44^{+}$population are more resistant to chemotherapy, we determined the changes of CD24 ${ }^{-} / \mathrm{CD} 44^{+}$ BCSCs after Dox treatment with or without ANXA3 knockdown (Fig. S4). Those results indicated that DOX treatment induced an increase trend of CD24-/CD44 $4^{+}$ BCSC population, which was eliminated by $A N X A 3$ depletion. These results suggested that the $A N X A 3$ knockdown enhanced the sensitivity of doxorubicin in breast cancer cells via increasing the cellular uptake, so the combination of $A N X A 3$ inhibition and doxorubicin therapy could inhibit tumor growth as well as tumor metastasis, which provides a novel combinational therapy approach to breast cancer.

\section{Discussion}

ANXA3, a membrane associated protein, has been reported to be abnormally expressed in various cancers.
$A N X A 3$ is highly expressed in lung, liver, and ovarian carcinoma and is correlated with a poor prognosis of patients $^{19-22}$. However, ANXA3 expression was essentially reduced in prostate and thyroid cancers, and the expression of $A N X A 3$ was negatively correlated with tumor development ${ }^{30-32}$. These studies suggest that $A N X A 3$ may play different roles in different tumors and reflect the heterogeneity and complexity of tumors ${ }^{33}$. However, $A N X A 3$ has not been well studied in breast cancer, and the biological role of ANXA3 is still unclear.

In this study, we found that $A N X A 3$ was significantly upregulated in breast cancer tissues at both the mRNA and protein levels, which were verified by qRT-PCR and immunohistochemical staining, respectively. $A N X A 3$ was also negatively correlated with patient prognosis in Kaplan-Meier survival analysis, which is consistent with a previous finding by Zeng et al. ${ }^{34}$.

In addition, we systematically studied the effect of $A N X A 3$ on breast cancer in vitro and in vivo, and this is the first investigation of the effect of $A N X A 3$ on BCSCs. Interestingly, others' previous findings showed that $A N X A 3$ could simultaneously promote tumor cell proliferation and invasion in lung and liver carcinoma ${ }^{25,35}$, but we found that 
ANXA3 knockdown inhibited tumor cell invasion but promoted cell proliferation both in vitro and in vivo. Our previous study showed the heterogeneity of BCSCs: the mesenchymal-like state $\left(\mathrm{CD} 24^{-} / \mathrm{CD} 44^{+}\right)$with a highly invasive capacity and the epithelial-like state $\left(\mathrm{ALDH}^{+}\right)$with an extensive ability to proliferate ${ }^{16}$. Currently, we showed that $A N X A 3$ knockdown induced MET and decreased the CD24 ${ }^{-} / \mathrm{CD}_{4} 4^{+}$population but increased the $\mathrm{ALDH}^{+}$ population. Furthermore, previous studies showed that ANXA3 can promote $\mathrm{CD}_{133^{+}}{ }^{\mathrm{CSC}} \mathrm{s}$ but there is no such meticulous distinction observed in the CSCs from lung or liver carcinoma, usually defined as the $\mathrm{CD} 133^{+}$population $^{25}$, so the divergence between our current study and others' studies in functional assays is understandable.

Next, we unraveled the mechanisms by which $A N X A 3$ knockdown induced the MET. Through gene expression profiling coupled with functional rescue experiments, we found that the NF- $\mathrm{kB}$ pathway was inhibited by ANXA3 knockdown. Multiple studies have shown that the NF- $\mathrm{KB}$ pathway is involved in the $\mathrm{EMT}^{36}$. Recent studies also found that NF- $\mathrm{KB}$ pathway activation regulates $\mathrm{CD} 133^{+}$ $\mathrm{CSCs}^{37-39}$ and $\mathrm{CD}^{3} 4^{+} \mathrm{CSCs}^{40,41}$, but no studies have reported about the effect of the NF- $\kappa B$ pathway on $\mathrm{ALDH}^{+}$CSCs. Our current study showed the NF-kB pathway regulation on the heterogeneity of BCSCs. Furthermore, I $\mathrm{KB} \alpha$-knockdown experiments showed effective but not complete rescue of the ANXA3-knockdown effect. Since, at the same time, we found that HRas and Wnt7b were also affected by the ANXA3 knockdown (Fig. 5a, b), the MAPK and Wnt pathways may also play a role in $\mathrm{BCSC}$ regulation by $A N X A 3$, but more work is needed to validate this hypothesis.

As ANXA3 is reported to assist in chemotherapy resistance $\mathrm{e}^{21,22,24}$, our study focused on the therapeutic potential of targeting $A N X A 3$. The increased cellular uptake of doxorubicin was observed in vitro when $A N X A 3$ was silenced. Considering that increased expression of annexin A1 and annexin A4 is also associated with drug resistance ${ }^{42,43}$, those members of the membrane-binding protein family may play important roles in chemotherapy resistance. Despite the fact that interfering with ANXA3 alone could significantly inhibit tumor metastasis with and cause increased tumor growth unexpectedly in vivo, targeting ANXA3 in combination with doxorubicin therapy could inhibit tumor growth and metastasis simultaneously. A study by Tong et al $^{25}$ showed that the original anti- $A N X A 3$ mouse antibody in combination with cisplatin exerted a synergistic inhibitory effect against hepatocellular carcinoma. Altogether, our results suggest that targeting ANXA3 may be a promising novel treatment for tumor therapy in combination with the doxorubicin chemotherapy.

In conclusion, our study showed that there is a negative correlation between ANXA3 expression and breast cancer prognosis. ANXA3 could affect breast cancer cell proliferation and invasion by regulating the transition between mesenchymal-like state BCSCs and epitheliallike state BCSCs via the NFKB pathway. A combination of targeting ANXA3 and chemotherapy could provide a promising therapeutic approach to inhibit tumor growth and metastasis.

\section{Materials and methods \\ Patients and clinical samples}

The human breast cancer tissue used in this study, obtained from the First Affiliated Hospital of Anhui Medical University (Hefei, Anhui, China), was comprised of 16 pairs of breast cancer tissues and their corresponding adjacent normal tissues (Table S1). For each tissue sample, a piece was embedded with paraffin for an IHC assay, and another piece was ground with liquid nitrogen and then lysed with TRIzol (Thermo Fisher Scientific, New York, USA) to extract the total RNA for real-time PCR assay.

\section{Cell culture}

Human breast cancer cell lines MDA-MB-231 and MDA-MB-468 and mouse breast cancer cell line 4T1 were purchased from ATCC. All of the cell lines were tested and authenticated shortly before use. These cell lines were cultured in RPMI-1640 medium with 10\% fetal bovine serum (Thermo Fisher Scientific, New York, USA) and $1 \%$ streptomycin/penicillin (Beyotime, Shanghai, China) and maintained in a $37{ }^{\circ} \mathrm{C}$ atmosphere with $5 \%$ carbon dioxide $\left(\mathrm{CO}_{2}\right)$.

\section{Short hairpin RNA plasmids and virus infection}

shRNA plasmids were purchased from Sigma-Aldrich (St. Louis, Missouri, USA), and the effective sequences of ANXA3, Anxa3, and I $\mathrm{B} \alpha$ used in this study are described in Table S2. Knockdown (KD) lentiviruses were prepared by transfecting $293 \mathrm{~T}$ cells; then, the cell lines were infected, and puromycin (Thermo Fisher Scientific, New York, USA) selection was performed for stable cell line establishment.

\section{RNA extraction and quantitative real-time PCR}

Total RNA was extracted with RNAiso Plus (Takara, Beijing, China) and RNA concentration was measured with NanoDrop (Thermo Fisher Scientific, New York, USA). Complementary DNA (cDNA) was prepared from $1 \mu \mathrm{g}$ RNA using the ReverTra Ace qPCR RT Kit (TOYOBO, Shanghai, China). qRT-PCR was carried out using AceQ qPCR SYBR Green Master Mix (Vazyme Biotech, Nanjing, China) in a real-time PCR system (7300, Applied Biosystems, New York, USA). TBP (TATA-box binding protein) was used as a reference gene. All primers used are shown in Table S3. 


\section{Western blotting}

Cells were collected and lysed in RIPA buffer containing a protease and phosphatase inhibitor cocktail (Roche, Mannheim, Germany) for $30 \mathrm{~min}$ on ice, and protein concentration was measured using a BCA Protein Assay Kit (Pierce, New York, USA). Then, samples mixed with 5x loading buffer were subjected to sodium dodecyl sulfate polyacrylamide gel electrophoresis. Proteins were transferred onto polyvinylidene fluoride (PVDF) membranes (Millipore, Billerica, USA) and incubated with the corresponding primary antibody and HRP-conjugated secondary antibody. The following antibodies and dilutions were used: anti-ANXA3 (1:200, sc-101885, Santa Cruz, Dallas, USA), anti-E-cadherin (1:1000, \#20874-1-AP, Proteintech, Rosemont, USA), anti-N-cadherin (1:1000, \#14215, CST, Danvers, USA), anti- $\gamma$-catenin $(1: 1000, \# 2309 \mathrm{~s}, \mathrm{CST})$, antiVimentin (1:1000, \#5741s, CST), anti-IкB $\alpha$ (1:1000, \#4812, CST), anti-phospho-p65 (1:1000, \#3033, CST), antitubulin (1:1000, HC101-02, TransGen Biotech, Beijing, China), HRP-conjugated goat anti-mouse IgG (1:5000, sc2005, Santa Cruz) and HRP-conjugated goat anti-rabbit IgG (1:5000, sc-2004, Santa Cruz). Chemiluminescent detection was performed using an ImageQuant LAS 4000 mini imaging system (GE, Fairfield, USA) with Western HRP Substrate (WBLUF0500, Millipore).

\section{MTT cell proliferation assay}

Cells were seeded in 96-well culture plates at a density of 300-500 cells per well and cultured for 3, 5, or 7 days. MTT (Sigma-Aldrich) was added to a final concentration of $0.5 \mathrm{mg} / \mathrm{mL}$, and the plates were incubated at $37^{\circ} \mathrm{C}$ for $4 \mathrm{~h}$. Then, $100 \mu \mathrm{L}$ Dimethyl sulfoxide (DMSO) per well was added after removing the supernatants and shaking the plate for $10 \mathrm{~min}$. The optical density at $490 \mathrm{~nm}\left(\mathrm{OD}_{490}\right)$ was measured with an Elx800 microplate reader (BioTek, Winooski, USA).

\section{Invasion assay}

Twenty-thousand cells were seeded in Matrigel-coated (354234, Corning, New York, USA) Transwell chambers ( $8 \mu \mathrm{M}$ Pore, 0216, BD, New York, USA), serum-free, with medium and 10\% FBS in the bottom well. After culturing for $36 \mathrm{~h}$, cells were fixed and stained with $0.1 \%$ crystal violet, and the invaded cells were photographed for statistical analysis.

\section{Flow cytometry}

For the ALDEFLUOR assay (StemCell Technologies, Cambridge, USA), dissociated cells were suspended in assay buffer containing ALDEFLUOR substrate and incubated with or without aldehyde dehydrogenase inhibitor DEAB. A CD24/CD44 assay was performed with anti-CD24 (1:20, 561647, BD) and anti-CD44 (1:100, 560532, BD). For analysis of tumorigenesis in tumor cell suspensions, anti- mouse-lineage antibodies were used for H2Kd (1:100, 116607, Biolegend), CD45 (1:50, 555483, BD), CD31 (1:50, 555446, BD), CD140b (1:50, 558821, BD), and CD235a (1:50, 555570, BD). A MoFlo Astrios instrument (Beckman Coulter, Brea, USA) was used, and data acquisition and analysis were performed using Summit software.

\section{In vivo tumorigenesis}

In this study, nude mice and BALB/c mice were utilized. All mice were bred and housed in AAALAC-accredited specific pathogen-free rodent facilities at the University of Science and Technology of China (Hefei, China). All mouse experiments were conducted according to standard operating procedures approved by the University Committee on the Use and Care of Animals at the University of Science and Technology of China. For the MDA-MB-231 tumorigenesis assay, $2 \times 10^{5}$ cells were injected into the fourth mammary gland of 6- to 8-weekold female nude mice. In the 4T1 experiment, $2 \times 10^{5}$ cells were injected into the fourth mammary gland of 6 - to 8-week-old female BALB/c mice, and doxorubicin was administered at $5 \mathrm{mg} / \mathrm{kg}$ via intraperitoneal injection weekly. Tumors were monitored weekly until mice were sacrificed when the diameter of tumors reached 1.0-1.5 $\mathrm{cm}$. Tumor volume was calculated as $1 / 2 \times$ length $\times$ width $^{2}$. For each tumor, one part was embedded in paraffin for histological analysis and the rest was digested into a single cell suspension using collagenase/hyaluronidase (Stem Cell Technologies) for flow cytometry. Lungs were embedded for $\mathrm{HE}$ staining and metastasis analysis.

\section{Immunohistochemistry}

The slices of paraffin-embedded tissues were dewaxed and rehydrated in xylene and graded alcohol solutions. Anti-ANXA3 (sc-101885, Santa Cruz) and anti-Ki67 (1:200, ZA-0502, ZSGB-BIO, Beijing, China) were used to stain the slices. Cell nuclei were stained with hematoxylin (ZLI-9610, ZSGB-BIO).

\section{Gene expression profiling}

Cells were collected, washed, and stored in RNAiso plus (Takara) at $-80{ }^{\circ} \mathrm{C}$, and gene expression profiling was performed at BGI (Shenzhen, China). Generally, after total RNA extraction and DNase I treatment, magnetic beads with Oligo(dT) were used to isolate mRNA, and the mRNA was fragmented into short fragments by mixing with the fragmentation buffer. cDNA was synthesized using the mRNA fragments as templates. After single nucleotide A (adenine) addition, the short fragments were connected with adapters. An Agilent 2100 Bioanalyzer and an ABI StepOnePlus Real-Time PCR System were used for quantification and qualification of the sample library. Finally, the library was sequenced using an 
Illumina $\mathrm{HiSeq}^{\mathrm{TM}} 2000$ platform or another sequencer when necessary.

\section{Statistical analysis}

All values are presented as the mean \pm s.e.m., except where otherwise indicated. Statistical analysis was performed using a two-tailed Student's $t$-test with GraphPad Prism 6 (GraphPad Software, La Jolla, USA). A $p$-value $<0.05$ was considered statistically significant.

The log-rank $p$-values of the overall survival analyses for breast cancer patients, the data of which was obtained from the Oncomine database (available at http://www. Oncomine.org), were computed using GraphPad Prism 6.

\section{Acknowledgements}

This work was supported by an NSFC grant (81530075), the National Key Research and Development Program of China (Stem Cell and Translationa Research 2016YFA0101202), NSFC grants (81472741 and 81322033), the MOST grant (2015CB553800), Fudan University Research Foundation IDH1340042, and the Research Foundation of the Fudan University Shanghai Cancer Center YJRC1603.

\section{Author details}

'The CAS Key Laboratory of Innate Immunity and Chronic Disease, Hefei National Laboratory for Physical Sciences at the Microscale, School of Life Science and Medical Center, University of Science \& Technology of China, Hefei, Anhui 230027, China. ${ }^{2}$ Key Laboratory of Breast Cancer in Shanghai, Cancer Institute, Department of Breast Surgery; Institutes of Biomedical Sciences; Innovation Center for Cell Signaling Network; Fudan University Shanghai Cancer Center, Shanghai 200032, China. ${ }^{3}$ The Department of Breast Surgery, The First Affiliated Hospital, Anhui Medical University, Hefei, Anhui 230032, China. ${ }^{4}$ Department of Neurosurgery, Anhui Provincial Hospital Affiliated to Anhui Medical University, Hefei, Anhui 230001, China. ${ }^{5}$ AnHui Province Key Laboratory of Brain Function and Brain Disease, Hefei, Anhui 230001, China

\section{Authors' contributions}

R.D.: conception, design, data analysis, interpretation and manuscript writing. B. L.: collection and assembly of data, data analysis and interpretation; L.Z., D.W. X.H., X.X., and L.Z.: collection and/or assembly of data and data analysis. C.N. and S.L.: conception, design, financial support, and manuscript writing.

\section{Conflict of interest}

The authors declare that they have no conflict of interest.

\section{Publisher's note}

Springer Nature remains neutral with regard to jurisdictional claims in published maps and institutional affiliations.

\section{Supplementary information}

The online version of this article (https://doi.org/10.1038/s41419-017-0143-z) contains supplementary material.

Received: 10 August 2017 Revised: 25 October 2017 Accepted: 10 November 2017

Published online: 26 January 2018

\section{References}

1. Siegel, R. L., Miller, K. D. \& Jemal, A. Cancer statistics, 2015. Ca. Cancer J. Clin. 65 5-29 (2015)

2. Nguyen, D. X., Bos, P. D. \& Massague, J. Metastasis: from dissemination to organ-specific colonization. Nat. Rev. Cancer 9, 274-284 (2009).
3. Tanei, T. et al. Association of breast cancer stem cells identified by aldehyde dehydrogenase 1 expression with resistance to sequential paclitaxel and epirubicin-based chemotherapy for breast cancers. Clin. Cancer Res. 15, 4234-4241 (2009).

4. Kalluri, R. \& Weinberg, R. A. The basics of epithelial-mesenchymal transition. J. Clin. Invest. 119, 1420-1428 (2009).

5. Thiery, J. P., Acloque, H., Huang, R. Y. \& Nieto, M. A. Epithelial-mesenchymal transitions in development and disease. Cell 139, 871-890 (2009).

6. Yang, J.\& Weinberg, R. A. Epithelial-mesenchymal transition: at the crossroads of development and tumor metastasis. Dev. Cell 14, 818-829 (2008).

7. Mallini, P., Lennard, T., Kirby, J. \& Meeson, A. Epithelial-to-mesenchymal transition: what is the impact on breast cancer stem cells and drug resistance. Cancer Treat. Rev. 40, 341-348 (2014).

8. Clevers, H. Wnt/beta-catenin signaling in development and disease. Cell 127, 469-480 (2006).

9. Grande, M. et al. Transforming growth factor-beta and epidermal growth factor synergistically stimulate epithelial to mesenchymal transition (EMT) through a MEK-dependent mechanism in primary cultured pig thyrocytes. $J$. Cell Sci. 115, 4227-4236 (2002).

10. Huber, M. A. et al. NF-kappaB is essential for epithelial-mesenchymal transition and metastasis in a model of breast cancer progression. J. Clin. Invest. 114, 569-581 (2004).

11. Song, W. et al. Expression and clinical significance of the stem cell marker CD133 in hepatocellular carcinoma. Int. J. Clin. Pract. 62, 1212-1218 (2008).

12. Bertolini, G. et al. Highly tumorigenic lung cancer CD133(+) cells display stemlike features and are spared by cisplatin treatment. Proc. Natl Acad. Sci. USA 106, 16281-16286 (2009).

13. Wang, Q. et al. Cancer stem cell marker CD133+ tumour cells and clinical outcome in rectal cancer. Histopathology 55, 284-293 (2009).

14. Ginestier, C. et al. ALDH1 is a marker of normal and malignant human mammary stem cells and a predictor of poor clinical outcome. Cell Stem Cell 1, 555-567 (2007).

15. Dean, M., Fojo, T. \& Bates, S. Tumour stem cells and drug resistance. Nat. Rev. Cancer 5, 275-284 (2005).

16. Liu, S. et al. Breast cancer stem cells transition between epithelial and mesenchymal states reflective of their normal counterparts. Stem Cell Rep. 2, 78-91 (2014).

17. Perron, B., LewitBentley, A., Geny, B. \& RussoMarie, F. Can enzymatic activity, or otherwise, be inferred from structural studies of annexin III? J. Biol. Chem. 272, 11321-11326 (1997).

18. Gerke, V., Creutz, C. E. \& Moss, S. E. Annexins: linking Ca2+ signalling to membrane dynamics. Nat. Rev. Mol. Cell Biol. 6, 449-461 (2005).

19. Liu, Y. F. et al. Quantitative proteomic analysis of metastasis-associated proteins in human primary lung adenocarcinoma. Prog. Biochem. Biophys. 36, 448-457 (2009).

20. Liu, Y. F. et al. Quantitative proteome analysis reveals annexin A3 as a novel biomarker in lung adenocarcinoma. J. Pathol. 217, 54-64 (2009).

21. Pan, Q. Z. et al. Annexin A3 promotes tumorigenesis and resistance to chemotherapy in hepatocellular carcinoma. Mol. Carcinog. 54, 598-607 (2015).

22. Yan, $X$. et al. Increased expression of annexin A3 is a mechanism of platinum resistance in ovarian cancer. Cancer Res. 70, 1616-1624 (2010).

23. Tong, S. W. et al. Proteomic investigation of 5 -fluorouracil resistance in a human hepatocellular carcinoma cell line. J. Cell. Biochem. 113, 1671-1680 (2012).

24. Penzvalto, $Z$. et al. Identifying resistance mechanisms against five tyrosine kinase inhibitors targeting the ERBB/RAS pathway in 45 cancer cell lines. PLoS ONE 8, e59503 (2013).

25. Tong, $M$. et al. ANXA3/JNK signaling promotes self-renewal and tumor growth, and its blockade provides a therapeutic target for hepatocellular carcinoma. Stem Cell Rep. 5, 45-59 (2015).

26. Pan, Q. Z. et al. Annexin A3 as a potential target for immunotherapy of liver cancer stem-like cells. Stem Cells 33, 354-366 (2015).

27. Al-Hajj, M., Wicha, M. S., Benito-Hernandez, A., Morrison, S. J. \& Clarke, M. F. Prospective identification of tumorigenic breast cancer cells. Proc. Natl Acad. Sci. USA 100, 3983-3988 (2003).

28. Jacobs, M. D. \& Harrison, S. C. Structure of an IkappaBalpha/NF-kappaB complex. Cell 95, 749-758 (1998).

29. Nolan, G. P., Ghosh, S., Liou, H. C., Tempst, P. \& Baltimore, D. DNA binding and I kappa B inhibition of the cloned p65 subunit of NF-kappa B, a rel-related polypeptide. Cell 64, 961-969 (1991). 
30. Wozny, W. et al. Differential radioactive quantification of protein abundance ratios between benign and malignant prostate tissues: cancer association of annexin A3. Proteomics 7, 313-322 (2007)

31. Kollerrmann, J. et al. Expression and prognostic relevance of annexin A3 in prostate cancer. Eur. Urol. 54, 1314-1323 (2008).

32. Jung, E. J. et al. Decreased annexin A3 expression correlates with tumo progression in papillary thyroid cancer. Proteom. Clin. Appl. 4, 528-537 (2010).

33. Gerlinger, M. et al. Intratumor heterogeneity and branched evolution revealed by multiregion sequencing. N. Engl. J. Med. 366, 883-892 (2012).

34. Zeng, $C$. et al. Annexin $\mathrm{A} 3$ is associated with a poor prognosis in breast cance and participates in the modulation of apoptosis in vitro by affecting the $\mathrm{BCl}-2$ / Bax balance. Exp. Mol. Pathol. 95, 23-31 (2013).

35. Liu, Y. F., Liu, Q. Q., Zhang, Y. H. \& Qiu, J. H. Annexin A3 knockdown suppresses lung adenocarcinoma. Anal. Cell. Pathol. 2016, 4131403 (2016).

36. Huber, M. A., Beug, H. \& Wirth, T. Epithelial-mesenchymal transition: NF-kappaB takes center stage. Cell Cycle 3, 1477-1480 (2004).

37. Long, $\mathrm{H}$. et al. Autocrine CCL5 signaling promotes invasion and migration of CD133+ ovarian cancer stem-like cells via NF-kappaB-mediated MMP-9 upregulation. Stem Cells 30, 2309-2319 (2012).
38. Annabi, B., Laflamme, C., Sina, A., Lachambre, M. P. \& Beliveau, R. A MT1MMP/NF-kappaB signaling axis as a checkpoint controller of COX-2 expression in CD133+ U87 glioblastoma cells. J. Neuroinflamm. 6, 8 (2009).

39. Li, K. et al. Downregulation of DNA-PKcs suppresses P-gp expression via inhibition of the Akt/NF-kappaB pathway in CD133-positive osteosarcoma MG-63 cells. Oncol. Rep. 36, 1973-1980 (2016).

40. Erdogan, S. et al. The flavonoid apigenin reduces prostate cancer CD44(+) stem cell survival and migration through PI3K/Akt/NF-kappaB signaling. Life Sci. 162, 77-86 (2016)

41. Kong, L. et al. Overexpression of SDF-1 activates the NF-kappaB pathway to induce epithelial to mesenchymal transition and cancer stem cell-like phenotypes of breast cancer cells. Int. J. Oncol. 48, 1085-1094 (2016).

42. Wang, Y. et al. Annexin-l expression modulates drug resistance in tumor cells. Biochem. Biophys. Res. Commun. 314, 565-570 (2004).

43. Han, E. K., Tahir, S. K., Cherian, S. P., Collins, N. \& Ng, S. C. Modulation of paclitaxel resistance by annexin IV in human cancer cell lines. Br. J. Cancer $\mathbf{8 3}$ 83-88 (2000). 